\title{
What could a new disease activity score for polymyalgia rheumatica do better?
}

\section{Burkhard F. Leeb (D)}

In their Review on monitoring and longterm management of giant cell arteritis (GCA) and polymyalgia rheumatica (PMR) (Camellino, D., Matteson, E. L., Buttgereit, F. \& Dejaco, C. Monitoring and long-term management of giant cell arteritis and polymyalgia rheumatica. Nat. Rev. Rheumatol. 16, 481-495 (2020) $)^{1}$, Camellino et al. considered the need for a new disease activity score for PMR, concluding that the established Polymyalgia Rheumatica Activity Score (PMR-AS) is seldom used in clinical practice or in clinical trials. To date, the publication in which the PMR-AS was first proposed ${ }^{2}$ has been cited 138 times according to Google Scholar, 13 of which were in 2020 , which gives testimony to its acceptance and implementation in scientific rheumatology.

The PMR-AS was originally derived from the EULAR response criteria for $\mathrm{PMR}^{3}$. Pain was recognized as such an important feature that it was chosen as the only parameter that had to decrease obligatorily in the PMR-AS, whereas at least three of the other four features, namely the physician's global assessment (PGA), C-reactive protein (CRP) level or erythrocyte sedimentation rate (ESR), upper limb elevation and morning stiffness, had to improve.

On the basis of an OMERACT study group publication $^{4}$ from 2017, Camellino et al. proposed that systemic inflammation detected by laboratory tests, physical function, pain and stiffness should be included in a new score for PMR monitoring ${ }^{1}$. This statement might surprise rheumatologists, as the individual components of the PMR-AS already cover all those domains.

Either a CRP level or an ESR can be used to calculate the PMR-AS, both of which are the acute-phase reactant tests predominantly used in clinical practice. One can debate whether upper limb elevation is the best measure of functionality, however, it is a cardinal symptom of PMR, and morning stiffness clearly covers the stiffness parameter. Of course, a discussion of how important the PGA is for a score's reliability is justified. However, other widely used scores include the PGA, which is often designated as the counterbalance to patient-related parameters ${ }^{5,6}$.
Cronbach's alpha (a measure of reliability) for the PMR-AS was between 0.91 and 0.88 in two patient cohorts $(>0.7$ indicates high reliability), and factorial analysis showed that all five single parameters contribute considerably to the overall result, with pain and PGA exerting the greatest influence ${ }^{2}$.

The PMR-AS has been applied in several studies $^{7,8}$ and comprises all the parameters proposed by Camellino et al. ${ }^{1}$ : why then should this score not be used for monitoring PMR in clinical practice or in clinical trials, or even be used as a surrogate for remission ${ }^{9}$ ? What should a new score be capable of that the PMR-AS is not? Using other parameters for function and stiffness, or leaving out the PGA, might change something, but pain and acute-phase reactants are not interchangeable and, ultimately, a new score must achieve the high internal consistency of the PMR-AS. Would developing a new score not be like the reinvention of the wheel?

There is a reply to this letter by Camellino, $\mathrm{D}$., Matteson, E. L., Buttgereit, F. \& Dejaco, C.
Nat. Rev. Rheumatol. https://doi.org/10.1038/ s41584-020-00551-5 (2020).

\section{Burkhard F. Leeb (iD) ${ }^{1,2}$ University Clinic for Internal Medicine - Rheumatology, St. Poelten, Austria. ${ }^{2}$ Karl Landsteiner Institute for Clinical Rheumatology, Hollabrunn, Austria. e-mail: leeb.rheuma@aon.at} https://doi.org/10.1038/s41584-020-00550-62021 Feb8

. Camellino, D., Matteson, E. L., Buttgereit, F. $\ltimes$ Dejaco, C. Monitoring and long-term management of giant cell arteritis and polymyalgia rheumatica. Nat. Rev. Rheumatol. 16, 481-495 (2020).

2. Leeb, B. F. \& Bird, H. A. A disease activity score for polymyalgia rheumatica. Ann. Rheum. Dis. 63 1279-1283 (2004).

3. Leeb, B. F. et al. EULAR response criteria for polymyalgia rheumatica: results of an initiative of the European Collaborating Polymyalgia Rheumatica Group (subcommittee of ESCISIT) Ann. Rheum. Dis. 62, 1189-1194 (2003).

4. Mackie, S. L. et al. The OMERACT core domain set for outcome measures for clinical trials in polymyalgia rheumatic. J. Rheumatol. 44, 1515-1521 (2017).

5. Smolen, J. S. et al. A simplified disease activity index for rheumatoid arthritis for use in clinical practice. Rheumatology 42, 244-257 (2003).

6. Aletaha, D. et al. Acute phase reactants add little to composite disease activity indices for rheumatoid arthritis: validation of a clinical activity score. Arthritis Res. Ther. 7, R796-R806 (2005).

7. Devauchelle-Pensec, V. et al. Assessing polymyalgia rheumatica activity when C-reactive protein is unavailable or uninterpretable. Rheumatology 57 , 666-670 (2018).

8. Manzo, C. \& Natale, M. Relapse of polymyalgia rheumatica after a fall. Reumatologia 55, 251-255 (2017).

9. Nothnagl, T. \& Leeb, B. F. Diagnosis, differential diagnosis and treatment of polymyalgia rheumatica. Drugs Aging 23, 391-402 (2006).

\section{Reply to: What could a new disease activity score for polymyalgia rheumatica do better?}

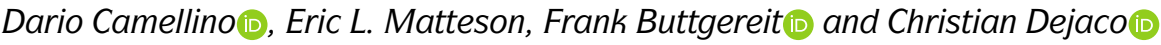

We thank Prof. Leeb for his interest in our Review (Camellino, D., Matteson, E. L., Buttgereit, F. \& Dejaco, C. Monitoring and long-term management of giant cell arteritis and polymyalgia rheumatica. Nat. Rev. Rheumatol. 16, 481-495 (2020)) $)^{1}$ and for his comments (Leeb, B. F. What could a new disease activity score for polymyalgia rheumatica do better? Nat. Rev. Rheumatol. https://doi.org/10.1038/s41584-020-00550-6 $(2020))^{2}$. As he points out, the Polymyalgia Rheumatica Activity Score (PMR-AS) is currently the only validated score for monitoring disease activity in patients with $\mathrm{PMR}^{3}$. Score calculation is straightforward, incorporating standard clinical data that captures disease features (including pain, stiffness, elevation of the upper limbs, physician's global assessment and inflammatory indexes) relevant for the evaluation of a patient with PMR.

Despite these advantages, the PMR-AS does not seem to be widely used. On 17 October 2020, we carried out a search of studies in patients with PMR through the Clinicaltrials. gov database. Among 105 studies retrieved, seven trials explicitly included the PMR-AS among their outcomes. We also analysed the publications included in the 2015 ACREULAR recommendations for the management of $\mathrm{PMR}^{4}$. Among the nine studies on 